\title{
Investigation into the Microscopic Mechanisms Influencing Convective Heat Transfer of Water Flow in Graphene Nanochannels
}

\author{
Drew C. Marable, Seungha Shin", and Ali Yousefzadi Nobakht \\ Department of Mechanical, Aerospace, and Biomedical Engineering, The University of \\ Tennessee, Knoxville, TN 37996, USA
}

\begin{abstract}
:
Convection heat transfer is assessed for laminarly flowing liquid water through graphene nanochannels via molecular dynamics (MD) simulations. The use of MD simulations allows for direct assessment of the minute details and mechanisms influencing overall heat transfer behaviors within our study; despite the presence of unrealistic axial conduction from temperature resetting and periodic boundary conditions within MD, hydrodynamically and thermally fully-developed water flow conditions are observed. It is indicated that the physics of convective heat transfer deviate from traditional macroscale theory as the no-slip boundary condition is violated with dimensional sizes descending towards the nanoscale; investigation into hydrodynamic slip and thermal slip, termed microscopic mechanisms, is performed for their influence on nanoscale convective outcomes. The parameters of graphene-water interaction strength, channel height, water velocity, and wall temperature are manipulated to evaluate resultant convection behaviors while comparing the effects of differing magnitudes of microscopic mechanisms imposed under various test conditions. This study finds microscopic interfacial mechanisms to significantly augment momentum and thermal behaviors and thus the conduct of convective heat transfer. Hydrodynamic and thermal slip are strongly correlated in all test case scenarios with the exception of velocity manipulation; the influence of thermal slip is found to dominate over that of hydrodynamic slip as surface advection is insignificant in high heat flux environments. Convective performance correlation is suggested as the ratio of thermal slip length to system size.
\end{abstract}

\section{Introduction}

The development of nanoscale electrical and mechanical devices has presented new challenges in thermal management and heat dissipation as these issues become important at smaller dimensional sizes [1]. The incorporation of nanochannels as a cooling method to efficiently remove excess heat is one avenue suggested for thermal management in these small scale devices [2-5]. Through previous works $[6,7]$, graphitic materials, and in particular the two-dimensional (2D) material graphene, has been identified as a material to improve microchannel thermal performance due to its excellent thermal conductivity, high surface-to-volume ratio, and ability to most effectively reduce the overall thermal resistance, as compared to that of more common silicon and aluminum substrates, when used as the primary substrate material of a microchannel heat sink [8]. Integration of graphene presents itself as a unique material for liquid cooling devices as $2 \mathrm{D}$ materials enable for the possibility of a single-layer channel wall and thus, effective heat dissipation devices at further reduced dimensional scales. In graphene-applied fluid cooling systems, graphene interlayer interactions are much weaker than intralayer interactions, therefore more emphasis and influence is placed on graphene-fluid interactions to dictate overall system performance.

The process of convection heat transfer in the macroscale realm has been thoroughly studied and is well established based on the no-slip boundary condition in which it is theorized that the fluid velocity at the wall should be equivalent to the wall velocity [9]. However, in contradiction to this theory, 
numerous studies have shown that at much smaller scales (or nanoscale) the no-slip boundary condition in liquids does not hold true and gives way to the development of slip flow as a result of surface effects dominating fluid flow behavior [10-19]. Furthermore, the convection heat transfer process, characterized by the dimensionless Nusselt number, $\mathrm{Nu}$, has been observed to deviate from that of traditional theory as dimension sizes decrease into the micro- and nanoscale realm $[4,15,20$ 23]. Reports indicate that temperature discontinuity (or temperature jump) at the wall due to interfacial thermal resistance decreases interfacial fluid temperature gradients and ultimately yields deviated convective behaviors from that of the macroscale [8, 23-26].

Although the study of nanoscale fluid flow characteristics and deviations from established theory have been extensively studied [16-19, 27], investigation into nanoscale convection heat transfer has been relatively unexplored. The study of sub-micrometer environments is necessary to fully understand momentum and heat transfer deviation from traditional theory [28]. Convection by monatomic molecules (e.g., Ar) has been the subject of a previous MD study [23]; however, the convective behaviors of complex liquid flows must be characterized and better understood within the nanoscale realm as liquid water is predominately employed as a liquid cooling media in many macroscale and microscale heat dissipation apparatus. In addition, convection in high-slip liquid fluid flows, as with 2D materials and increasingly hydrophobic interfaces, must be examined to improve understandings of momentum and energy transport within nanoscale systems [28].

Therefore, the behaviors and underlying mechanics within the nanoscale convective heat transfer process of liquid water, a complex fluid, flowing through nanochannels with double-layer graphene (DLG) acting as the channel walls are evaluated using non-equilibrium molecular dynamics (NEMD) techniques utilizing the LAMMPS package [29]; the majority of simulations relied on Extreme Science and Engineering Discovery Environment (XSEDE) resources [30]. The effects of various fluid-wall interaction strengths, channel heights, water velocities, and wall temperatures, in addition to their subsequent interfacial phenomena, are discussed.

\section{Methodology}

\subsection{Simulation Details}

Nanochannels consist of two parallel walls held into position using weak harmonic springs at a specified $z$-axis spacing, with each wall of the nanochannel being composed of double-layer graphene (DLG). From initial assessments of single-, double-, and triple- layer graphene for viable system wall composition, DLG is selected for its ability to ensure that the fluid-interacting wall is not directly thermostated, which can potentially result in molecular movement distortions and the reduction of data accuracy, as in the case of single-layer graphene. Moreover, in addition to increased computational efficiency over that of triple-layer graphene, DLG is chosen as multiple interlayer interactions are excluded to allow for a more direct focus on interfacial interactions and their subsequent effects.

The TIP4P/2005 [31] model is employed for the simulation of liquid water due to its predictable replication of ambient bulk water properties [32] with $\mathrm{O}-\mathrm{H}$ bond length of $0.09572 \mathrm{~nm}, \mathrm{H}-\mathrm{O}-\mathrm{H}$ bond angle of $104.52^{\circ}$, and charges of $\mathrm{O}\left(-1.04 e_{c}\right)$ and $\mathrm{H}\left(0.52 e_{c}\right)$, where $e_{c}$ is the charge of an electron. Here, a dummy atom (or massless charge site) $\mathrm{M}$ is introduced $0.01546 \mathrm{~nm}$ away from the $\mathrm{O}$ atom to improve electrostatic distribution around the water molecule. In addition to electrostatic forces, the dispersion/repulsion force is considered for intermolecular interactions and is modeled as the 12-6 Lennard-Jones (LJ) potential mode: 


$$
U_{L J}=4 \varepsilon\left[\left(\frac{\sigma}{r}\right)^{12}-\left(\frac{\sigma}{r}\right)^{6}\right],
$$

where $\varepsilon$ and $\sigma$ are the $\mathrm{LJ}$ interaction energy strength and distance at which the potential energy is zero, and $r$ is the distance between two particles. For the dispersion/repulsion force between water molecules, only O-O interactions are considered with parameters of $\varepsilon_{0-0}=0.7748 \mathrm{~kJ} / \mathrm{mol}$ and $\sigma_{0-0}=$ $0.3159 \mathrm{~nm}$. Water molecules are kept rigid, maintaining bond length $(\mathrm{O}-\mathrm{H})$ and angle $(\mathrm{H}-\mathrm{O}-\mathrm{H})$ constant using the SHAKE algorithm [33]. Liquid water molecules corresponding to a density of $\rho=$ $1000 \mathrm{~kg} / \mathrm{m}^{3}$ are placed in-between the two nanochannel walls; an additional $0.25 \mathrm{~nm}$ of spacing between the block of liquid water molecules and each nanochannel surface is added so to account for the water unoccupied volume [34-36].

Intralayer carbon interactions are modelled with the Tersoff-Brenner [37] potential, and interlayer carbon interactions are modeled by the 12-6 LJ potential with parameters from Ref. [38]. Watercarbon interactions are also described by the 12-6 LJ potential, where only the interactions between the carbon and oxygen atoms are defined so to increase computational efficiency; LJ interactions between carbon and hydrogen atoms were not introduced as these types of interactions are deemed negligible [39]. Moreover, previous investigations [40, 41], in which interactions between carbon and hydrogen atoms are neglected by modelling interactions between the water molecules and graphene atoms via dispersion (or van der Waals) forces, also employ this method of accounting for all watergraphene interactions by the $\mathrm{LJ}$ carbon-oxygen potential. The $\mathrm{LJ}$ values characterizing carbon-oxygen interactions were selected as $\varepsilon_{\mathrm{c}-\mathrm{o}}=0.4763 \mathrm{~kJ} / \mathrm{mol}$ and $\sigma_{\mathrm{c}-\mathrm{o}}=0.319 \mathrm{~nm}$ to obtain the most recent experimental water contact angle on a contaminant-free graphitic surface [42-44]. The cut-off distance for all Lennard-Jones interactions is $r_{c}=1.0 \mathrm{~nm}$.

The Verlet algorithm [45] is employed to integrate Newton's equations of motion within the simulation domain. In an effort to increase computational efficiency, periodic boundary conditions are applied to the $x$ - and $y$-axis directions while the $z$-axis is fixed as non-periodic. The particle-particle particle-mesh (PPPM) technique [46] is utilized to compute Coulombic interactions. The 2D-slab method [47] is employed to prevent Coulombic computations in the $z$-axis by adding a vacuum space and an electrostatic layer correction above and below the simulation domain to exclude wall-wall interactions across the $z$-axis boundary.

A channel height of $H=5.0 \mathrm{~nm}$ is chosen for the nanoscale evaluation of convection heat transfer. To ensure that fully-developed laminar flow inside the nanochannel is achieved, the corresponding channel length, $L$, for this height is determined through the inverse Graetz number, $G z^{-1}=$ $L /\left(D_{H} R e P r\right)$, as a function of the Reynolds number, $R e$, Prandtl number, $P r$, and hydraulic diameter, $D_{H}=4 A_{c} / P=2 H$, where $A_{c}$ is the cross-sectional area and $P$ is the wetted perimeter. $\operatorname{Re}$ and $\operatorname{Pr}$ were determined for each test case and calculated using characteristic lengths $(2 H)$ and the various required properties that were independently found from separate simulations (e.g., viscosity, thermal conductivity, etc.); properties were verified for consistency with previous reports and traditionally accepted values [31, 48, 49]. $R e$ was found to be in the range of $R e=0.065-1.21$ and $\operatorname{Pr}$ was determined as roughly $\mathrm{Pr}=4.8$. Statistical (or continuum) behavior of nanoscale fluid flow is expected to influence thermal and hydrodynamic development; in our system, this is characterized through the dimensionless Knudsen number, $K n=\lambda / D_{H}$, as a ratio of the molecular mean free path, $\lambda$, to a characteristic length set as the hydraulic diameter. Since a liquid is employed as the working fluid within our simulations, we approximate the mean free path of liquid water as the average intermolecular distance between two water molecules as $\delta \sim \lambda \sim\left(V_{m} / N_{A}\right)^{1 / 3} \sim 0.3 \mathrm{~nm}$, where $V_{m}$ is the molar volume and $N_{A}$ is Avogadro's number [50, 51]; thus, we find our system Knusden $(K n, \sim 0.03)$ 
number value to be neither fully in the free molecular nor continuum regime [52, 53]. In addition to the possibility of size effects rending the no-slip boundary condition, based on continuum NavierStokes approximations, difficult to predict and/or invalid at the nanoscales [54-56], we hypothesized that continuum fluid behaviors may be observed with considerable influences from microscopic mechanisms (the potential presence of slip flow). From this, we anticipate potential slip flow behavior to require a larger channel entry length than of the length determined from setting $G z^{-1}=0.05$ for the continuum regime; hence, to ensure full flow development inside the nanochannel, a large value of $G z^{-1}=0.4$ is chosen $[51,57,58]$. Moreover, considering the potential effects of axial conduction as well as the areas of the liquid pumping and temperature rescaling regions, the respective channel length is found to be $L=12.0 \mathrm{~nm}$. It must be noted that we do not claim for our molecular system (and others later described) to fully or statistically satisfy the aforementioned continuum based nondimensional numbers, however to a certain extent, they do have the ability to be representative of similar physical description of continuum fluid behavior and is evidenced in the following sections by the observance of resulting laminar flows and the formation of fully developed velocity and temperature profiles. A channel width of $W=3.0 \mathrm{~nm}$ is selected and does not vary across all simulations; this value is chosen for computational efficiency as it was found that an increased channel width $(W=4.0,5.0$, and $6.0 \mathrm{~nm})$ produced no influence on resulting convective behaviors.

\subsection{Fluid Flow Method}

Flow simulations typically utilize periodic boundary conditions coupled with a constant flowdriving body force in the $x$-direction, $f_{b, x}$, applied to each water molecule; this allows for the liquid to exit one side of the periodic box (outlet) and re-enter across the periodic boundary (inlet). However, due to the additional energy of the body force and the presence of a temperature gradient within the channel, the energy between the inlet and outlet is not conserved, thereby distorting the convective heat transfer process. In order to effectively simulate convective heat transfer, the thermal pump method $[23,59]$ is introduced to the fluid domain. The thermal pump method divides the fluid domain into three separate regions: a forcing region, a temperature reset region, and a data collection region, as depicted in Fig. 1.

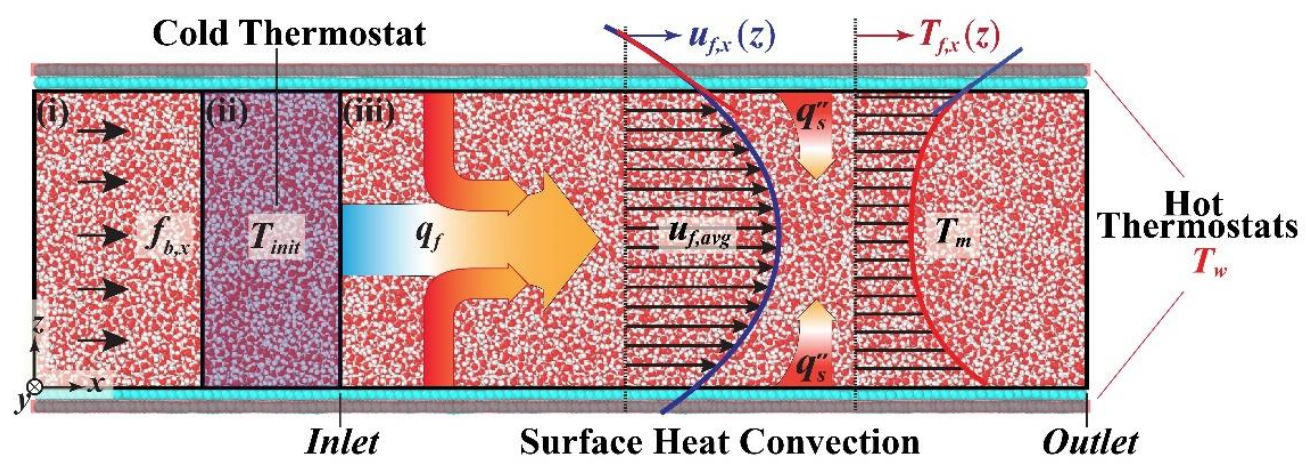

Figure 1. Schematic of the simulation domain separated into three regions: (i) forcing region, (ii) temperature rescaling region, and (iii) data collection region.

The forcing region is applied to the $x$-directional coordinates of $0.0 \mathrm{~nm}<x<0.8 \mathrm{~nm}$ and is kept constant for all simulations; the water molecules inside this region experience a constant body force of $f_{b, x}$ to drive flow. Temperature resetting is performed only for water molecules inside the region of 0.8 $\mathrm{nm}<x<1.4 \mathrm{~nm}$; as with the forcing region, these $x$-directional coordinates are held constant for all simulations and test cases. In order to reset the water inlet temperature while maintaining the average velocity of water, thermal velocities are rescaled after removing the streaming velocity bias from the forcing region and then the previously removed streaming velocity bias is added back to the liquid 
water molecules. After the water molecules undergo temperature rescaling, molecules enter into the data collection region in which the convective heat transfer process is observed with water flowing from the inlet at a specified initial temperature and heated by the thermostated walls. Simulations are performed for a total duration of $7.0 \mathrm{~ns}$ using a $1.0 \mathrm{fs}$ time step. An initial equilibration phase is implemented in the $N V T$ ensemble (constant number of particles, volume, and temperature) $[60,61]$ for $1.0 \mathrm{~ns}$ to thermally relax the system. After the relaxation period (i.e., negligible fluctuation in $T$ and $u_{f}$ profiles), further simulation is carried out for $6.0 \mathrm{~ns}$ in the $N V E$ microcanonical ensemble (constant number of atoms, volume, and energy) to update the velocities and coordinates of the fluid atoms; in this simulation period, the outer-most graphene walls are heated to specified constant thermostated temperature, $T_{w}$, while only the specified fluid domain area is rescaled to a constant inlet temperature, $T_{\text {init }}$, using Langevin thermostats [62].

The assessment of convection heat transfer is evaluated by means of collecting and analyzing velocity and temperature distributions, in addition to heat flux through the water-interacting walls. Velocity and temperature data are collected into equally distributed bins across the $x$ - and $z$-axis directions while surface heat flux data in the $z$ direction is recorded at similarly spaced channel subsections only along the $x$-axis (or flow) direction. Simulation data is collected over the simulation duration, after relaxation.

The use of thermostats to control temperature in MD simulations, as an artificial procedure of energy addition and extraction within systems, has the potential to cause unrealistic system behaviors. However, as shown in Fig. 2, velocity distributions of the fluid agree well with the MaxwellBoltzmann velocity distribution at a temperature of $300 \mathrm{~K}$; thus, validating our use of the thermal pump method to effectively simulate the convective heat transfer process.

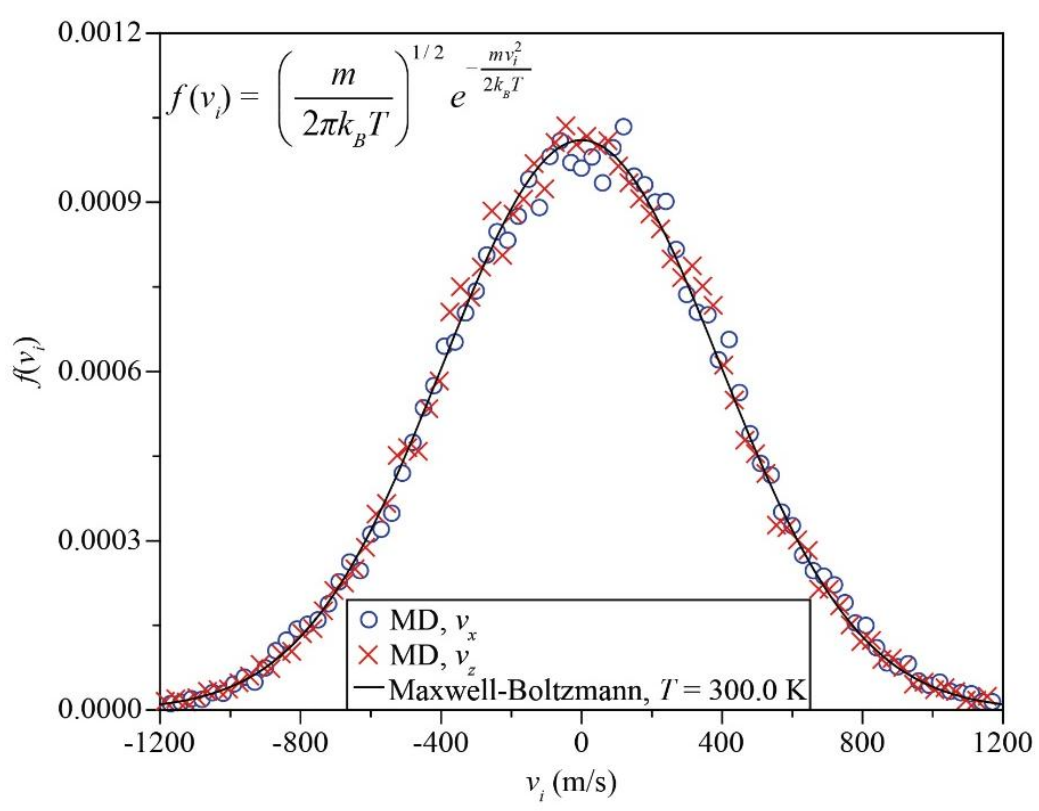

Figure 2. Maxwell-Boltzmann (MB) velocity distributions. The blue open circles and red cross markers represent that of the $x$ - and $z$-axis distributions from MD, respectively; the black solid line corresponds to the MB distribution curve at $300 \mathrm{~K}$.

\subsection{Governing Equations}

The convective heat transfer process is evaluated based on the local heat transfer coefficient, $h(x)$, at a $x$-directional position according to: 


$$
h(x)=\frac{q_{s}^{\prime \prime}(x)}{T_{w}-T_{m}(x)},
$$

where $q_{s}$ "' $(x)$ is the local heat flux across the channel surface (interface between water and graphene, $\left.\mathrm{W} / \mathrm{m}^{2}\right), T_{w}$ is the wall temperature, and $T_{m}(x)$ is the local mean temperature at $x$, defined as:

$$
T_{m}(x)=\frac{\int_{0}^{H} c \rho(z) u_{f, x}(z) T(x, z) d z}{\int_{0}^{H} c \rho(z) u_{f, x}(z) d z},
$$

where $H$ is the channel height, $c$ is the specific heat capacity and is assumed to be constant, $\rho$ is the fluid density, $u_{f, x}(z)$ is the fluid velocity is the $x$-direction, and $T(x, z)$ is the fluid temperature along the $x$-axis. Using the local average velocity and kinetic energy obtained from MD allows for the determination of $u_{f, x}(z)$ and $T(x, z)$. The heat flux across the channel surface is calculated as [63]:

$$
q_{s}^{\prime \prime}=\frac{Q}{A_{s} \Delta t}=\frac{1}{V}\left\{\sum_{i} e_{i} u_{i, z}+\sum_{i<j}\left[\boldsymbol{f}_{i j} \cdot\left(\boldsymbol{u}_{i}+\boldsymbol{u}_{j}\right)\right] z_{i j}\right\},
$$

where $Q$ is the total energy that passes through the non-thermostated walls, $A_{S}$ is the wall surface area interacting with water, $\Delta t$ is the elapsed time, $V$ is the volume of the simulation domain, $e_{i}$ is the internal energy of the atom, $u_{i, z}$ is the $i$-th atom velocity in the $z$ direction, $\boldsymbol{u}_{i}$ and $\boldsymbol{u}_{j}$ are the velocities of atoms $i$ and $j, f_{i j}$ is the pairwise force vector between different atoms, and $z_{i j}$ is the directional position vector between atoms $i$ and $j$. Traditional determination of convection heat transfer by internal flow is characterized through the non-dimensional Nusselt number, $N u_{D}$, as a ratio of convection to conduction normal to the interface. By combining Eqs. (2), (3), and (4), we evaluate $N u_{D}$ as:

$$
N u_{D}=\frac{h D_{h}}{k_{f}}=\frac{2 H q_{s}^{\prime \prime}}{k_{f}\left[T_{w}-T_{m}(x)\right]},
$$

where $k_{f}$ is the thermal conductivity of the fluid. Without advection effects, $q_{s}$ " is equivalent to water conduction heat flux in the $z$ direction at the channel surface; i.e., $q_{s}{ }^{\prime \prime}=-\left.k_{f}(\partial T / \partial z)\right|_{z=0} . h$ and $N u_{D}$ are also calculated by using the conduction heat flux based on the temperature gradient at the wall as:

$$
\begin{aligned}
& h=\left.\frac{k_{f}}{T_{w}-T_{m}(x)} \frac{\partial T}{\partial z}\right|_{z=0}, \quad \text { and } \\
& N u_{D}=\frac{h D_{h}}{k_{f}}=\left.\frac{2 H}{T_{w}-T_{m}(x)} \frac{\partial T}{\partial z}\right|_{z=0} .
\end{aligned}
$$

The use of these two methods allow for comparative evaluation of nanoscale convective phenomena by accounting for heat flux across the channel surface, interfacial water conduction, and heat changes due to local advection. In addition, hydrodynamic and thermal slip at the wall are used to characterize and better identify underlying microscopic mechanisms contributing to the behavior of convection heat transfer within tested systems. The hydrodynamic slip length is utilized to identify the flow behaviors within each system and characterized as:

$$
l_{s}=\frac{\Delta u_{f, w / f}}{\left.\left(\frac{\partial u_{f, x}}{\partial z}\right)\right|_{z=0}}
$$

where $l_{s}$ is the slip length, $\Delta u_{f, w / f}$ is the difference between the fluid velocity at the wall and the wall velocity $\left(u_{w}=0 \mathrm{~m} / \mathrm{s}\right)$, or slip velocity, and $\left.\left(\partial u_{f, x} / \partial z\right)\right|_{z=0}$ is the velocity gradient in the fluid. Similar to 
the hydrodynamic slip length, fluid thermal behaviors at the interface are identified by the thermal slip length to relate the effect of temperature jump at the interface and interfacial thermal resistance, or Kapitza resistance, according to:

$$
l_{k}=k_{f} R_{w / f}=\frac{\Delta T_{w / f}}{\left.\left(\frac{\partial T}{\partial z}\right)\right|_{z=0}},
$$

where $l_{k}$ is the thermal slip length, $R_{w / f}$ is the interfacial thermal (Kapitza) resistance, $\Delta T_{w / f}$ is the difference between the fluid temperature at the wall and the wall temperature or the fluid temperature jump at the wall, and $\left.(\partial T / \partial z)\right|_{z=0}$ is the fluid temperature gradient at the wall. Extrapolating a quadratic function to the wall allows for the determination of $\Delta T_{w / f}$ and $\left.(\partial T / \partial z)\right|_{z=0}$.

\subsection{Control Parameters}

Previous reports $[4,23,64]$ indicate that microscopic mechanisms, characterized by hydrodynamic and thermal slip at the interface, alter channel flow characteristics at smaller scales to yield deviated convective behaviors from that of the theoretical macroscale $N u_{D}$ value for fully-developed laminar flow in-between two parallel plates at constant wall temperature [65, 66]. Varying magnitudes of microscopic mechanisms are expected to emerge as flow characteristics are known to differ with wall wettability $\left(\varepsilon_{\mathrm{c}-\mathrm{o}}\right)$, channel height $(H)$, water velocity $\left(u_{f, a v g}\right)$, and wall temperature $\left(T_{w}\right)$. Therefore, the influence of hydrodynamic and thermal slip on subsequent nanoscale convection heat transfer behaviors are assessed for the imposed test conditions of $\varepsilon_{\mathrm{c}-\mathrm{o}}, H, u_{f, a v g}$, and $T_{w}$.

Characterized by the wetting contact angle on the wall surface, the influence of wall wettability is performed by means of controlling the $\mathrm{LJ} \varepsilon_{\mathrm{c}-\mathrm{o}}$ parameter [67]. Manipulation of this parameter allows for tuning the graphene wall to become either more hydrophobic or hydrophilic; decreasing the $L J \varepsilon_{c-0}$ value leads to reduced water-carbon interaction and produces to more hydrophobicity, while an increase in $\varepsilon_{\mathrm{c}-\mathrm{o}}$ value enhances interactions to yield more hydrophilicity. Selected values $\varepsilon_{\mathrm{c}-\mathrm{o}}$ of 0.1869 , 0.2834, 0.392 (Werder et al. [48]), 0.4763, 0.5728, 0.6693, $0.7748\left(\varepsilon_{\mathrm{c}-\mathrm{o}}=\varepsilon_{\mathrm{o}-\mathrm{o}}\right)$, and $1.0553 \mathrm{~kJ} / \mathrm{mol}$ were found to represent contact angles of $135^{\circ}, 114^{\circ}, 91^{\circ}, 68^{\circ}, 42^{\circ}, 18^{\circ}, 0^{\circ}$, and $0^{\circ}$, respectively, to assess varying magnitudes of hydrophobic and hydrophilic graphene wall interactions with water.

Channel heights in the range of $H=4.0$ to $8.0 \mathrm{~nm}$ are chosen to assess convection heat transfer size dependence in nanochannels; this range of channel heights was selected so to ensure the appearance of regions with constant bulk density of water and for computational affordability. Moreover, systems with channel heights of less than $4.0 \mathrm{~nm}$ were found to be unstable and produce unreliable data; we attribute instabilities in this range to large temperature and pressure gradients imposed by the thermal pump method in these smaller volumes. In the same manner as determining the initial dimensions of $12.0 \times 3.0 \times 5.0 \mathrm{~nm}^{3}$, Knusden numbers for the range of selected channel heights are also in the slip flow regime with values of $K n=0.0375,0.0300,0.0250,0.0214,0.0188$ for channel heights of 4.0, 5.0, $6.0,7.0$, and $8.0 \mathrm{~nm}$, respectively. Thus, we maintain a constant $G z^{-1}=0.4$ for each channel height to obtain the resulting dimensions of $8.0 \times 3.0 \times 4.0 \mathrm{~nm}^{3}, 12.0 \times 3.0 \times 5.0 \mathrm{~nm}^{3}, 16.0 \times 3.0 \times 6.0 \mathrm{~nm}^{3}$, $22.0 \times 3.0 \times 7.0 \mathrm{~nm}^{3}$ and $28.0 \times 3.0 \times 8.0 \mathrm{~nm}^{3}$ and correspond to $3282,6117,9897,15736$, and 22959 water molecules included in the system, respectively. Due to the varying amount of water molecules in each channel height simulation, the added flow-driving body force is specifically chosen for each channel height to ensure that the resulting water velocities are a constant $20 \mathrm{~m} / \mathrm{s}$ across all tests of channel height variations; this is implemented for accurate comparison and evaluation of results.

The water flow velocity inside the graphene nanochannel is generated by means of applying a flowdriving body force to the forcing region of the liquid pump setup. The magnitude of the flow-driving 
force is incrementally increased from a minimum value of $9.6 \times 10^{-15} \mathrm{~N} /$ molecule to a maximum value of $5.6 \times 10^{-14} \mathrm{~N} /$ molecule in order to observe linearly increasing average water velocity $\left(u_{f, a v g}\right)$ from 10 $\mathrm{m} / \mathrm{s}$ to $105 \mathrm{~m} / \mathrm{s}$, respectively.

Wall temperature effects on the convective heat transfer process within nanochannels are observed by increasing the thermostated wall temperature from a minimum temperature of $T_{w}=350 \mathrm{~K}$ to a maximum temperature of $T_{w}=1000 \mathrm{~K}$. Wall heating via thermostats is only applied to the outer-most graphene sheets so that the potential effects of direct thermostating on the fluid-interacting wall will not cause distortions in the molecular movements of water.

In order to allow for a common test case across all four investigated areas, unless otherwise stated simulation parameters are as follows: LJ interaction energy strength and distance are specified as $\varepsilon_{\mathrm{c}-\mathrm{o}}$ $=0.4763 \mathrm{~kJ} / \mathrm{mol}$ and $\sigma_{\mathrm{c}-\mathrm{o}}=0.319 \mathrm{~nm}$, respectively, channel dimensions are kept constant as $12.0 \times 3.0 \times 5.0 \mathrm{~nm}^{3}$ to correspond to 6117 water molecules filling the nanochannel, a flow-driving body force of $1.52 \times 10^{-14} \mathrm{~N} / \mathrm{molecule}$ is applied to the molecules in the forcing region which equates to a water velocity of approximately $20 \mathrm{~m} / \mathrm{s}$ for these dimensions, the inlet temperature is held constant at $T_{\text {init }}=300 \mathrm{~K}$, and the walls are thermostated to a temperature of $T_{w}=400 \mathrm{~K}$. The tested control parameters are summarized in Table 1. Moreover, the amount of error is expected to be low with the level of uncertainty ranging from roughly $3 \%$ to $5 \%$, which is estimated from three separate simulations for three different test cases $(H=4.0,5.0$, and $6.0 \mathrm{~nm})$.

Table 1. Simulation test case parameters.

\begin{tabular}{c|c|c|c|c}
\hline \hline Control Parameters & $\varepsilon_{\mathrm{c}-\mathrm{o}}(\mathrm{kJ} / \mathrm{mol})$ & $H(\mathrm{~nm})$ & $u_{f, a v g}(\mathrm{~m} / \mathrm{s})$ & $T_{w}(\mathrm{~K})$ \\
\hline Control Range & $0.1869-1.0553$ & $4.0-8.0$ & $10.0-105.0$ & $350.0-1000.0$ \\
\hline Constant* & 0.4763 & 5.0 & 20.0 & 400.0 \\
\hline \hline
\end{tabular}

*Common simulation among all test cases

\section{Results and Discussion}

\subsection{Velocity and Temperature Development}




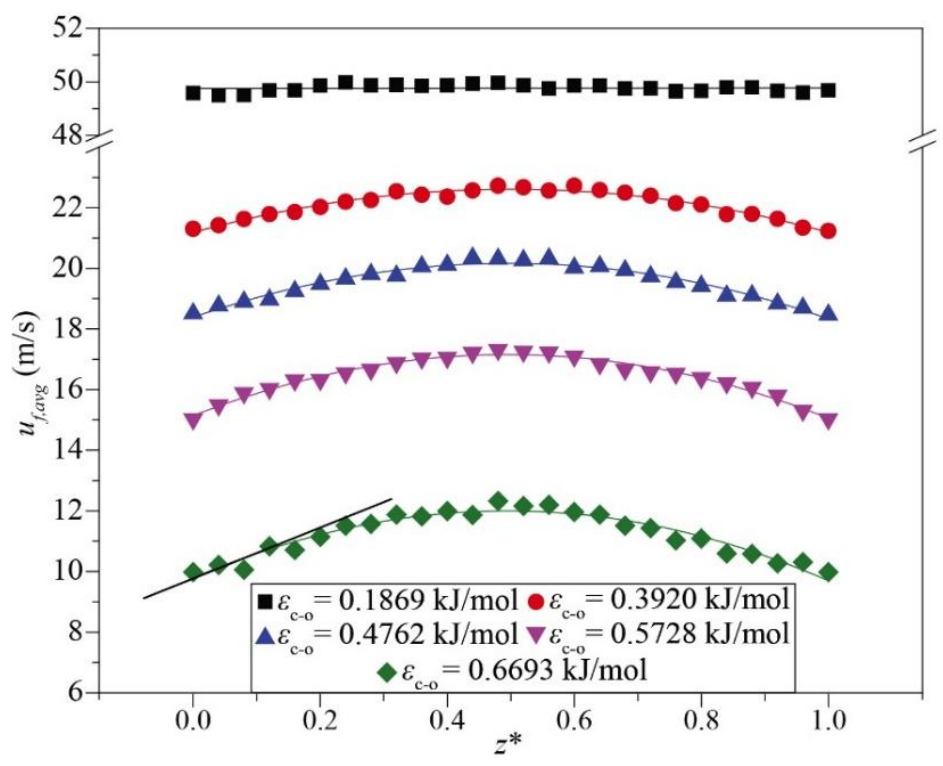

Figure 3. Velocity distribution across the dimensionless channel height $\left(z^{*}=z / H\right.$, and $\left.H=5.0 \mathrm{~nm}\right)$ with various wall-fluid interaction strengths $\left(\varepsilon_{\mathrm{c}-\mathrm{o}}\right)$. With increasing $\varepsilon_{\mathrm{c}-\mathrm{o}}$, average velocity decreases while the parabolic curvature increases.

Resultant velocity and temperature distributions from MD are utilized to describe hydrodynamic and thermal development within simulated systems for the characterization of convection heat transfer. In each system setup, hydrodynamic flow fields are formed as water molecules exit the liquid pump and temperature rescaling regions; velocity distributions are determined from MD and in all test cases the flow is laminar with $R e$ on the order of 1 . As expected, velocity distributions remain invariant in the flow direction, which gives indication that fully-developed hydrodynamic conditions have been achieved. Fully-developed flow characteristics are observed to vary when manipulating the parameters of $\varepsilon_{\mathrm{c}-\mathrm{o}}, H, u_{f, x}$, and $T_{w}$; most noticeable are changes in flow behavior and average velocity distribution for differing magnitudes of wettability (Fig. 3). In this case, lower interaction strengths (more hydrophobic) result in a high velocity plug-like flow with little distribution curvature, whereas increasing $\varepsilon_{\mathrm{c}-\mathrm{o}}$ (more hydrophilic) possess a more parabolic velocity distribution curvature and exhibit lower average velocities. In analyzing velocity distributions and as portrayed in Fig. 3, slip flow behavior is present within all simulated test cases; slip effects and their influences on system behavior will be discussed later. 
Water temperature distributions characterize thermal development inside the nanochannels as cold $\left(T_{\text {init }}=300 \mathrm{~K}\right)$ water leaves the inlet and flows past the heated walls; temperature distributions are the primary avenue used for the evaluation of convection heat transfer within the various system parameter configurations. Thermal development for each tested case is illustrated by its resulting isotherm, mean temperature distribution, and temperature evolution at various points along the $x$-axis as in Fig. 4; for visualization purposes, a nanochannel with dimensions of $22.0 \times 3.0 \times 7.0 \mathrm{~nm}^{3}$ is chosen for depiction. While typically neglected in macrochannels, axial conduction is clearly present within the liquid water as shown in Figs. 4a and 4b. To an extent, the nature of our employed simulation method can induce unrealistic axial conduction as a temperature gradient will be imposed across the system domain due to water molecules interacting across the periodic image. However, while physical influences of the simulation setup and procedure must also be considered, the presence and magnitude of axial conduction is largely facilitated by the fluid flow and heat transfer behaviors of the nanoscale liquid media [68]. Therefore, the influence of axial conduction on thermal development is considered for all test cases within this assessment of the nanoscale convection process.

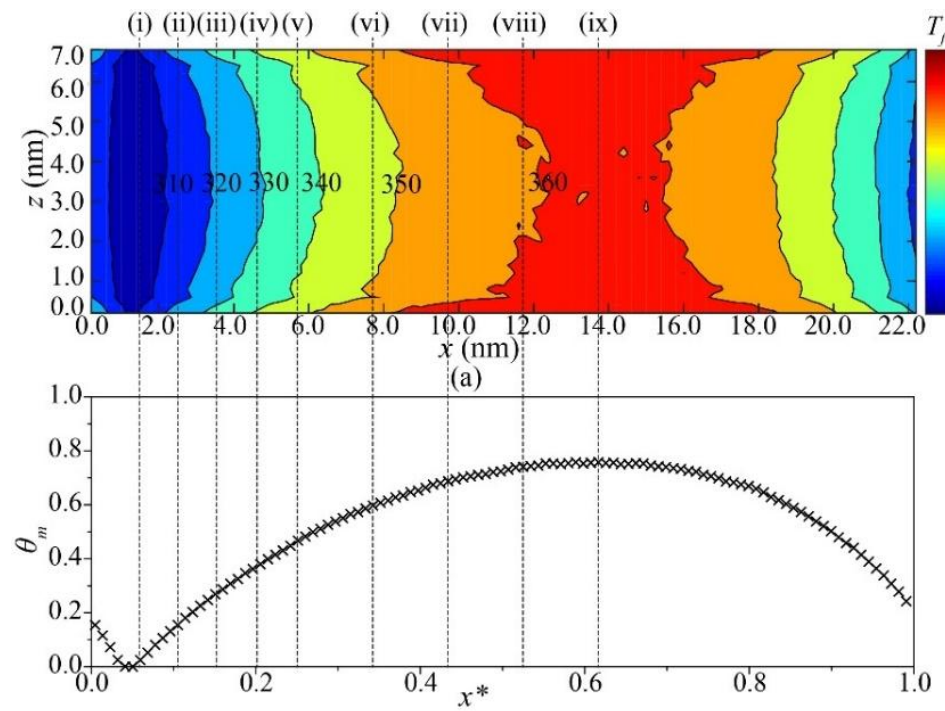

(b)

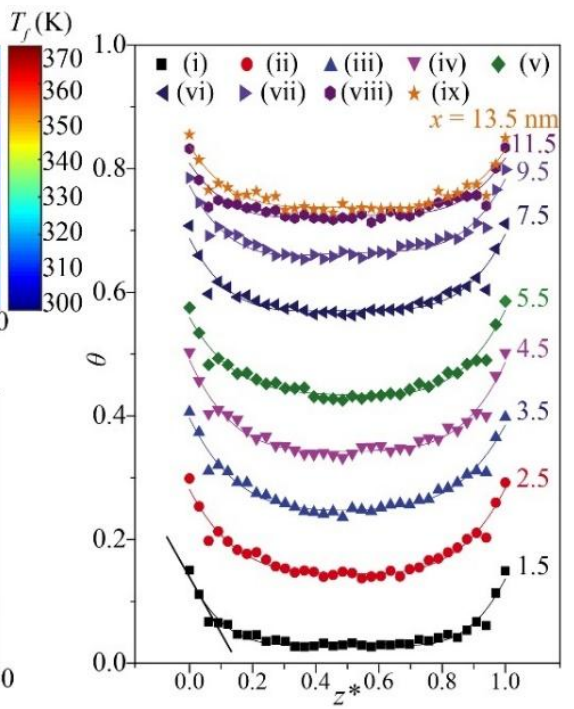

(c)

Figure 4. Temperature development inside a nanochannel with dimensions of $22.0 \times 3.0 \times 7.0 \mathrm{~nm}^{3}$. (a) Isotherm diagram of temperature development (isothermal lines are inverted to better guide the eye.) (b) Non-dimensional mean temperature $\left(\theta_{m}\right)$ development in the $x$-axis flow direction. (c) Development of non-dimensional $(\theta)$ temperature profiles across the channel height (z-axis) at various $x$-directional points in the direction of the flow.

Axial conduction can arise in instances of low $R e$ and $P r$, where thermal diffusion is more important than advection due to small inertia and high thermal diffusivity, to effectively distort the development of mean fluid temperature. Therefore, the significant parameter is the Peclet number, $P e=R e P r$; in laminar flows, previous reports indicate that axial conduction emerges at $P e<10$ and its influence decreases as $P e$ increases in micro and nanochannels $[58,68]$. Since $P e$ numbers range from 0.8 to 6.0 for the varying parameters of $\varepsilon_{\mathrm{c}-\mathrm{o}}, H, u_{f, x}$, and $T_{w}$, axial conduction is indicated to be a present factor within all test cases. Thus, a decrease in mean fluid temperature downstream is expected due to the influence of axial conduction and the temperature rescaling region (low $T$ ) across the periodic image. Figure 5a, showing the dimensionless $T_{m}\left[\theta=\left(T-T_{\text {init }}\right) /\left(T_{w}-T_{\text {init }}\right) ; \theta=\theta_{m}\right.$ when $\left.T=T_{m}\right]$ with respect to dimensionless channel distance from the entrance $\left(x^{*}=x / L\right)$ for varying $P e$, confirms the lessening influence of axial conductions influence within nanochannels at higher $\mathrm{Pe}$. Despite the presence of unrealistic effects, the fluid temperature gradient at the surface decreases from the inlet and trends to a flattened profile that displays little deviation as in Fig. 5b; this indicates that the internal flow is 
thermally fully developed. Although observation limitations within thermal development of internal flow may be present, this MD approach allows for the analysis of fully-developed convection behavior.

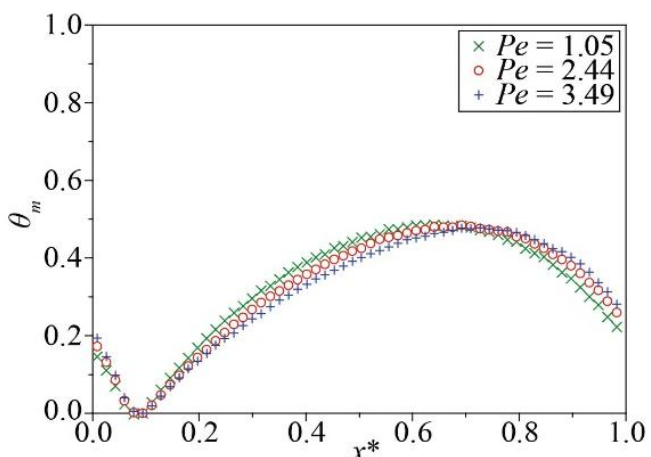

(a)

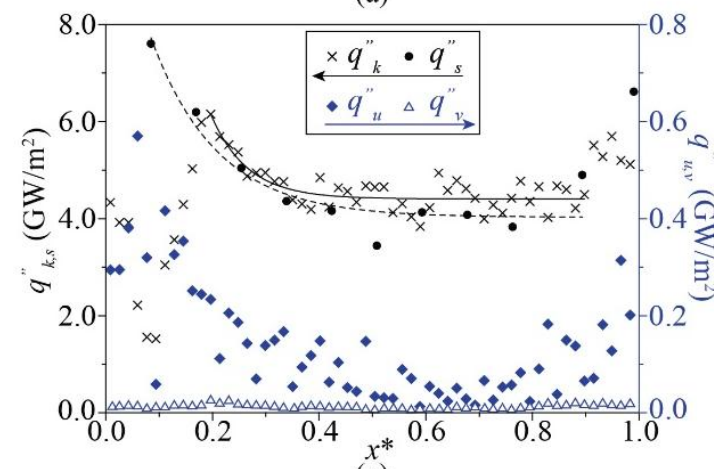

(c)

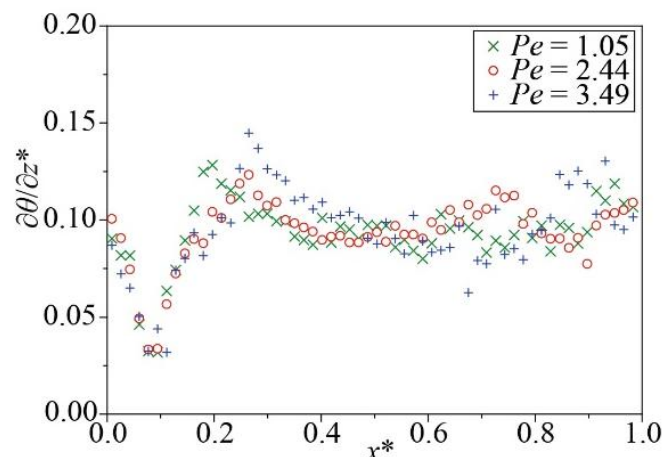

(b)

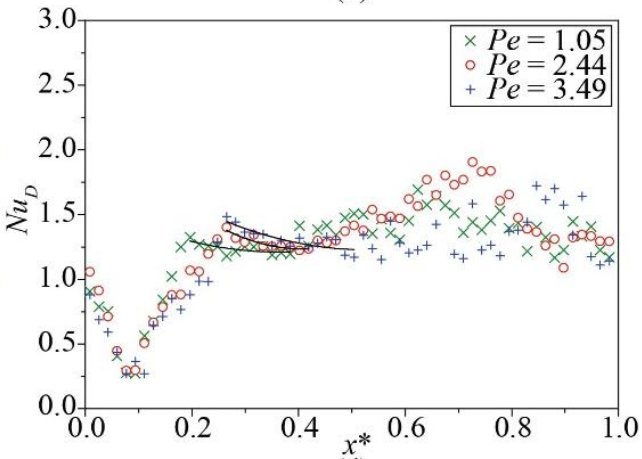

(d)

Figure 5. Flow development with axial conduction at different Peclet numbers showing (a) mean dimensionless temperature $\left(\theta_{m}\right)$ development, (b) dimensionless temperature gradient $\left(\partial \theta / \partial z^{*}\right)$ at the wall, (c) comparison of local surface $\left(q_{s}\right)$, surface water conduction $\left(q_{k}\right)$, change of surface advection $\left(q_{u}\right)$, and viscous $\left(q_{v}\right)$, heat flux and (d) local Nusselt number $\left(N u_{D}\right)$.

Local heat flux $\left(q_{s}\right.$ ") values must be determined according to Eq. (5) in order to evaluate the local Nusselt number $\left(N u_{D}\right)$ that characterizes convection. When utilizing the no-slip boundary condition in macroscale analysis, interfacial water conduction, as determined from the surface temperature gradient $\left[q_{k} "=-\left.k_{f}(\partial T / \partial z)\right|_{z=0}\right]$, can replace surface heat flux $\left(q_{s}{ }^{\prime \prime}\right)$. However, slip flow exists in all tested systems. Thus, the supplied heat from the wall $\left(q_{s}{ }^{\prime \prime}\right)$ is unable to be replaced by interfacial conduction $\left(q_{k}\right.$ ") as slip flow contributions facilitate an increase in advection heat flux $\left(q_{u}{ }^{\prime}\right)$ due to non-zero flow velocity at the surface, as in Fig. 3. $q_{u}{ }^{\prime \prime}$ is calculated from the surface fluid velocity (or slip velocity, $\left.u_{f, s}\right)$ and temperature change $\left(\Delta T_{f / w}\right)$ with an approximation of constant $\rho$ and $c\left(q_{u}=\right.$ $\left.\rho u_{f, s} c \Delta T_{f / w}\right)$. Figure $5 \mathrm{c}$ shows that $q_{u}$ " is at least one order of magnitude smaller than both $q_{k}$ " and $q_{s}$, therefore $q_{u}$ " is negligible in the data collection region for fully-developed $N u_{D}$ calculations. As prescribed simulation conditions in MD induce large amounts of heat, heat flux with respect to viscous dissipation, $q_{v}{ }^{\prime \prime}=\mu(\partial u / \partial z)^{2}$ where $\mu$ is the dynamic viscosity of the water and is found from Ref. [69], is insignificant to influence $q_{k}$ " or $q_{s}$ " and can be ignored. Figure 5c also shows close agreement between the determinations of $q_{k}$ " and $q_{s}$ " with a difference of only $9.32 \%$; this comparatively small difference is attributed to negligible $q_{u}$ " and $q_{v}$. Additionally, variations in water thermal conductivity near the interface are anticipated to influence the $q_{k}$ "calculation, however this contribution is expected to be minor as $q_{k}$ " and $q_{s}$ " are in relative agreement [70]. Therefore, due to the close agreement of heat flux calculation approaches and the computationally expensive procedure of determining localized heat fluxes $\left(q_{s}\right.$ ) within small regions, $q_{k}$ " is employed for calculations of the local Nusselt number in Fig. 5d. This method also displays the axial conduction's effect on the local 
Nusselt number in which $N u_{D}$ trend profiles are almost flat at lower $\mathrm{Pe}$; conversely, higher $\mathrm{Pe}$ values give way to more exponential and traditional $N u_{D}$ trends. Here, we focus on fully-developed convection behaviors, rather than flow development in the entry region, which are properly characterized by the local Nusselt number, $N u_{D, f d}$, due to attenuated axial conduction, as illustrated in

Fig. 5d. Average $N u_{D}$ can also be calculated using $\overline{N u}_{D}=(1 / k) \int_{0}^{L} h(x) d x$, as it approaches that of local fully-developed $N u_{D}$ if the channel length is sufficiently long. However, as inclusion of entrance regions under the influence of unrealistic axial conduction may distort resulting values for average $N u_{D}$ (due to the limited simulation cell size), we report fully-developed Nusselt numbers, $N u_{D f f}$, for all test cases in the following sections.

\subsection{Microscopic Mechanisms}

In addition to hydrodynamic and thermal development, velocity and temperature distributions are employed to enhance clarity and understanding of microscopic mechanisms influencing simulated system behavior. Characterizing these mechanisms, or microscopic interfacial interactions, allows for hydrodynamic and thermal slip lengths, Eqs. (7) and (8), respectively, to be considered for their influence of system outcomes within convection assessments. Hydrodynamic slip length provides information describing fluid flow behavior, the potential effects of surface advection and friction heating, and slip flow magnitude; the thermal slip length relays the significance of temperature jump and Kapitza resistance to system performance. In Fig. 6, we report deviation from the no-slip boundary condition in all tested simulation cases with the emergence of hydrodynamic and thermal slip at the interface as characterized by $l_{s}$ and $l_{k}$, respectively. In consistency with our water volume definition, although there is a small amount of uncertainty, the first liquid layer or slab closest to the wall was defined as the liquid-solid boundary [71, 72] with Fig. 6 displaying omission of the water unoccupied volume. We acknowledge that a previous report indicates [73] hydrodynamic slip length to be independent from the shear rate in the linear regime. From this, we clarify that although our findings are seemingly in contrast to the mentioned reference, we attribute reported changes in hydrodynamic slip length to manipulation of our control parameters. 


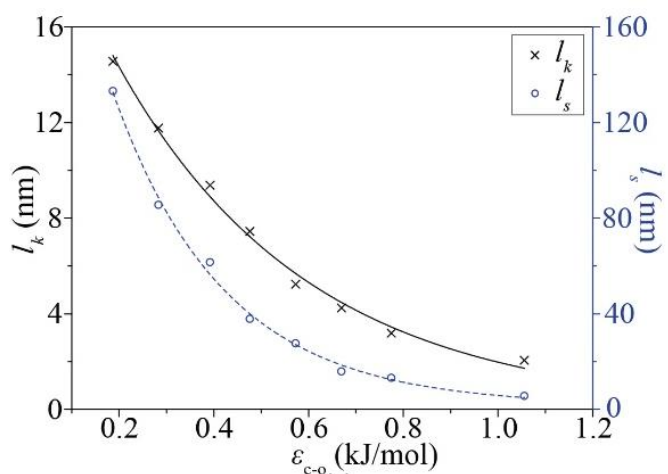

(a)

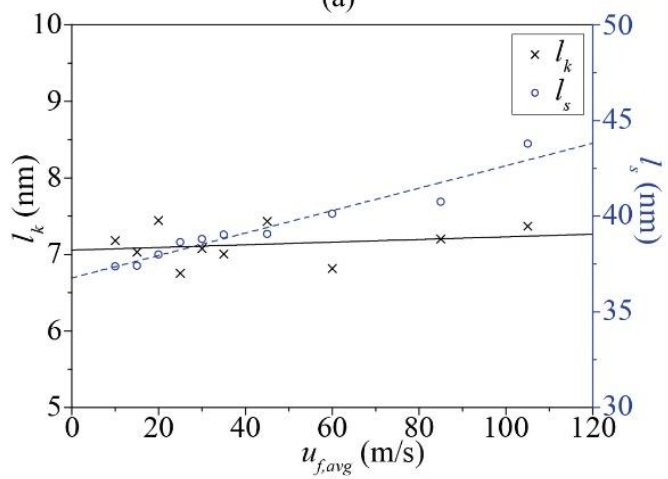

(c)

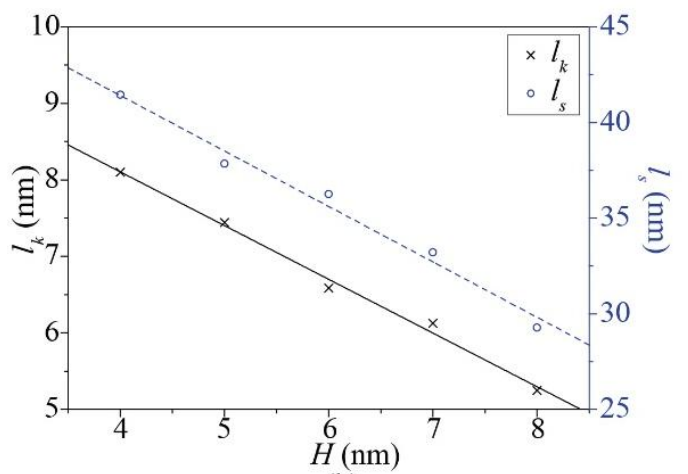

(b)

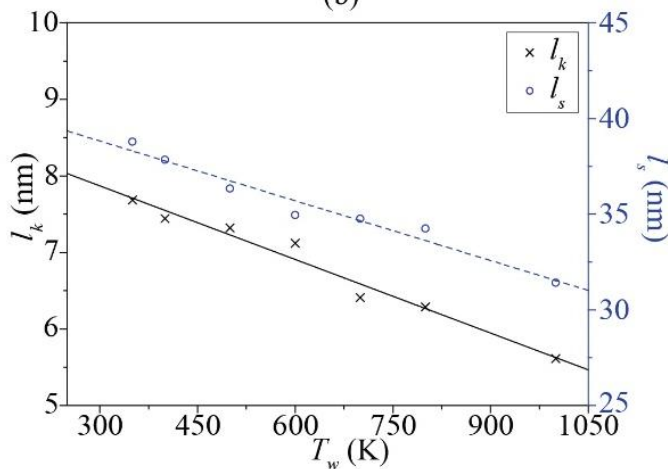

(d)

Figure 6. Thermal $\left(l_{k}\right)$ and hydrodynamic $\left(l_{s}\right)$ slip lengths for varying (a) interaction strengths $\left(\varepsilon_{\mathrm{c}-\mathrm{o}}\right)$, (b) channel heights $(H)$, (c) velocities $\left(u_{f, a v g}\right)$, and (d) wall temperatures $\left(T_{w}\right)$.

Among all tested parameters, varying wall wettability produces the most dramatic changes in hydrodynamic and thermal slip lengths as in Fig. 6a. As a result of increasing hydrophilicity due to larger surface interaction strengths, and thus increased friction at the interface [74], hydrodynamic slip at the boundary decreases from $l_{s}=133.17 \mathrm{~nm}$ for highly hydrophobic interactions to $l_{s}=5.64 \mathrm{~nm}$ for highly hydrophilic interactions. Likewise, Kapitza resistance is seen to follow in a similar manner with thermal slip lengths exponentially decreasing from 14.55 and $2.05 \mathrm{~nm}$ for the largest variations of $\varepsilon_{\mathrm{c}-\mathrm{o}}$; we note that this observation of thermal slip length exponentially varying with liquid-solid interaction strength is consistent with previous reports [75, 76], however further assessment should be performed to validate this exponential trend in nanoscale liquid flows. This behavior stems from a larger accommodation of water molecules and stronger coupling with the wall interface as interfacial interactions increase; therefore, temperature jump at the wall and thus, $R_{w / f}$ will decrease to allow for more effective heat transfer. Even with increasing interaction strengths past that at which results in a WCA of $0^{\circ}\left(\varepsilon_{\mathrm{c}-\mathrm{o}}=0.7748\right.$ and $\left.1.0553 \mathrm{~kJ} / \mathrm{mol}\right)$ the no-slip boundary condition is still violated; this is attributed to the atomically smooth nature of $2 \mathrm{D}$ graphene sheets acting at the channel walls [72].

In Fig. 6b, an increase in channel height from $H=4.0$ to $8.0 \mathrm{~nm}$ is shown to yield decreasing hydrodynamic slip lengths from $l_{s}=41.45$ to $29.27 \mathrm{~nm}$; this observation is consistent with a previous report [77] that attributes this behavior to the lessening effects of microscopic mechanisms at the interface projecting their influence on fluid behavior as dimensional scales increase. In comparable form, a similar decrease is observed for thermal slip length from $l_{k}=8.10$ to $5.24 \mathrm{~nm}$ at the same range of channel heights. We further the referenced suggestion and infer this to also be true with regard to thermal behaviors; the significance of temperature jump at the wall and Kapitza resistance will cease to interfere with heat transfer behaviors as size trends towards macroscale. 
In concurrence with previous reports [71, 78, 79], due to larger momentum difference at the liquidsolid boundary, hydrodynamic slip length increases with higher water velocities from $l_{s}=37.37$ to $43.78 \mathrm{~nm}$ for the channel velocities of $u_{f, a v g}=10$ and $105 \mathrm{~m} / \mathrm{s}$, respectively. However, unlike all other thermal slip length trends in other test cases, $l_{k}$ with respect to changing velocity is seen to remain relatively constant at $7.13 \pm 0.228 \mathrm{~nm}$ in Fig. 6c. While an increase in thermal slip length is expected as with hydrodynamic slip length, we propose that momentum changes at the surface due to increasing velocity are not significant enough to produce noticeable effects on the resulting thermal diffusion within the channel. To further support this claim, channel velocities are significantly smaller than calculated thermal velocities on the order of $680 \mathrm{~m} / \mathrm{s}$ for resulting simulation water temperatures; therefore, it is inferred that manipulation of channel velocity will produce no meaningful change in thermal slip length until the fluid media velocity is on the order of its thermal velocity.

A decrease in hydrodynamic slip length from $l_{s}=38.78$ to $31.41 \mathrm{~nm}$ is observed for increasing wall temperatures from 350 to $1000 \mathrm{~K}$ (Fig. 6d). This is contrary to conventional knowledge in which it might be assumed that higher fluid temperatures will yield increased water velocities, and thus increased slip lengths. While average velocities (at the same magnitude of prescribed flow-driving force) are seen increase from 18.47 to $34.17 \mathrm{~m} / \mathrm{s}$ for $T_{w}=350$ and $1000 \mathrm{~K}$, respectively, this behavior is attributed to the fact that as temperature increases, atom kinetic energy and thus momentum transfer due to collisions will become enhanced to allow for decreasing slip lengths [80]. Similarly, enhanced heat transfer due to increased collisions at higher temperatures will allow for decreased Kapitza resistance [81] and thus more effective diffusion of heat from the wall to the water. Subsequently, with increasing thermostated wall temperature from $T_{w}=350 \mathrm{~K}$ to $T_{w}=1000 \mathrm{~K}$ thermal slip lengths are observed to decrease from $l_{k}=7.68$ to $5.61 \mathrm{~nm}$.

\subsection{Fully-Developed Heat Transfer}

Due to their negligible influence within macroscale convection analysis, the microscopic mechanisms of hydrodynamic and thermal slip at the interface are unaccounted for. Conversely, as dimensions decrease and efforts are made to assess nanoscale thermal analysis, microscopic mechanisms must be considered for their role within the convection process as their influence becomes magnified and projected on overall heat transfer performance. Subsequently, for all simulated test cases the resulting behavior of convection heat transfer displays reduced Nusselt values from that of the theoretical macroscale fully-developed laminar flow, $N u_{D f f}=7.541$, as seen in Fig. 7. The presence and magnitude of hydrodynamic and thermal slip at the interface are shown to impede heat transfer from the graphene wall to the water and dictate the extent of convection effectiveness. 


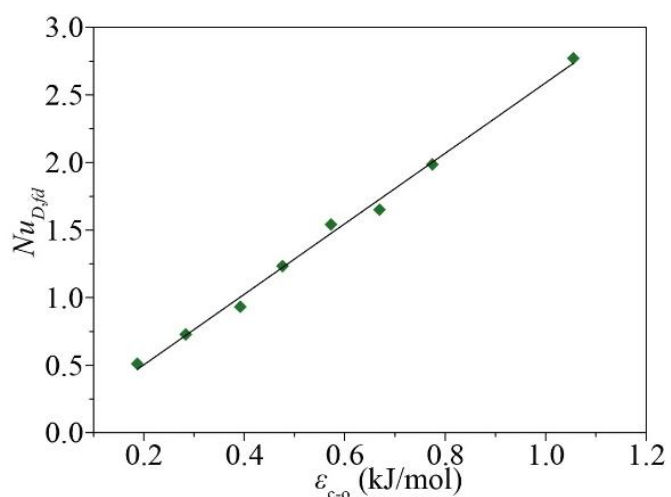

(a)

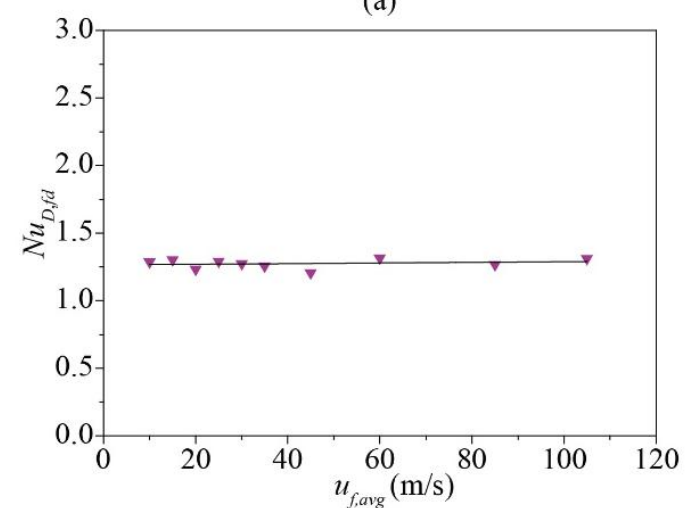

(c)

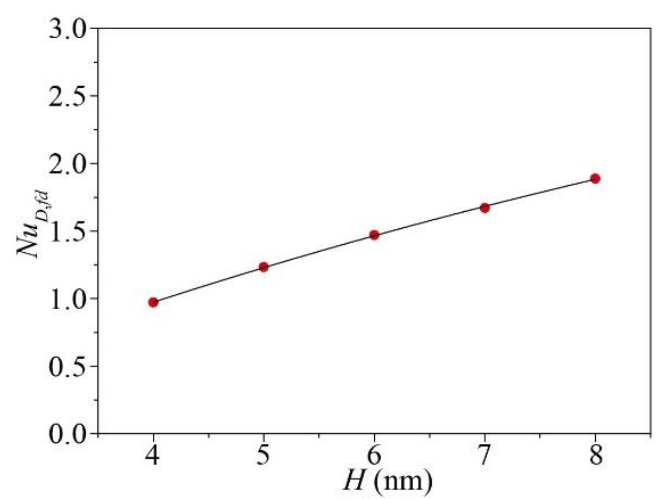

(b)

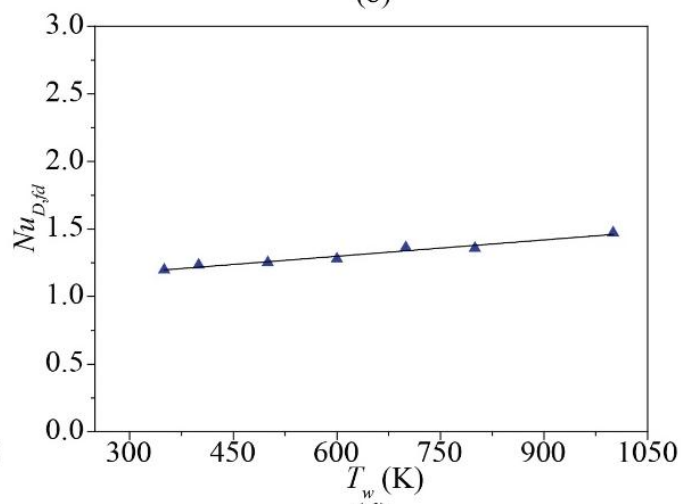

(d)

Figure 7. Fully-developed Nusselt number $\left(N u_{D f f d}\right)$ trends for varying (a) interaction strength $\left(\varepsilon_{\mathrm{c}-\mathrm{o}}\right)$, (b) channel height $(H),(\mathrm{c})$ water velocity $\left(u_{f, \text { avg }}\right)$, and $(\mathrm{d})$ wall temperature $\left(T_{w}\right)$.

Manipulation of the water-graphene surface interaction strength yields significant enhancement to the convection heat transfer process as wall wettability is tuned from hydrophobic to hydrophilic (Fig. $7 \mathrm{a})$; our results are consistent with previous reports $[4,23]$ which indicate that the microscale convection process will become enhanced as increased interactions between the wall and water allow for improved heat transfer. Results show an order of magnitude increase in heat transfer performance from $N u_{D f f}=0.594$ for the case of extreme hydrophobicity $\left(\varepsilon_{\mathrm{c}-\mathrm{o}}=0.1869 \mathrm{~kJ} / \mathrm{mol}\right)$ to $N u_{D}=2.771$ corresponding the extreme hydrophilicity case $\left(\varepsilon_{\mathrm{c}-\mathrm{o}}=1.0553 \mathrm{~kJ} / \mathrm{mol}\right)$. This conduct stems from stronger interactions allowing more water molecules to come into contact with the wall, thus inhibiting momentum and advection effects at the interface as illustrated by decreasing hydrodynamic slip lengths. Likewise, thermal slip lengths are seen to decrease because of more wall-fluid interactions enhancing heat transfer at the surface as more water molecules are available to remove heat from the wall, which is in agreement with previous findings [16, 82]. Density profiles for corresponding $\varepsilon_{\mathrm{c}-\mathrm{o}}$ values in Fig. 8, which are similar to that of another report [34], displays this increasing water molecule density near the interfacial regions that enable for enhanced heat transfer at higher interaction strengths. 


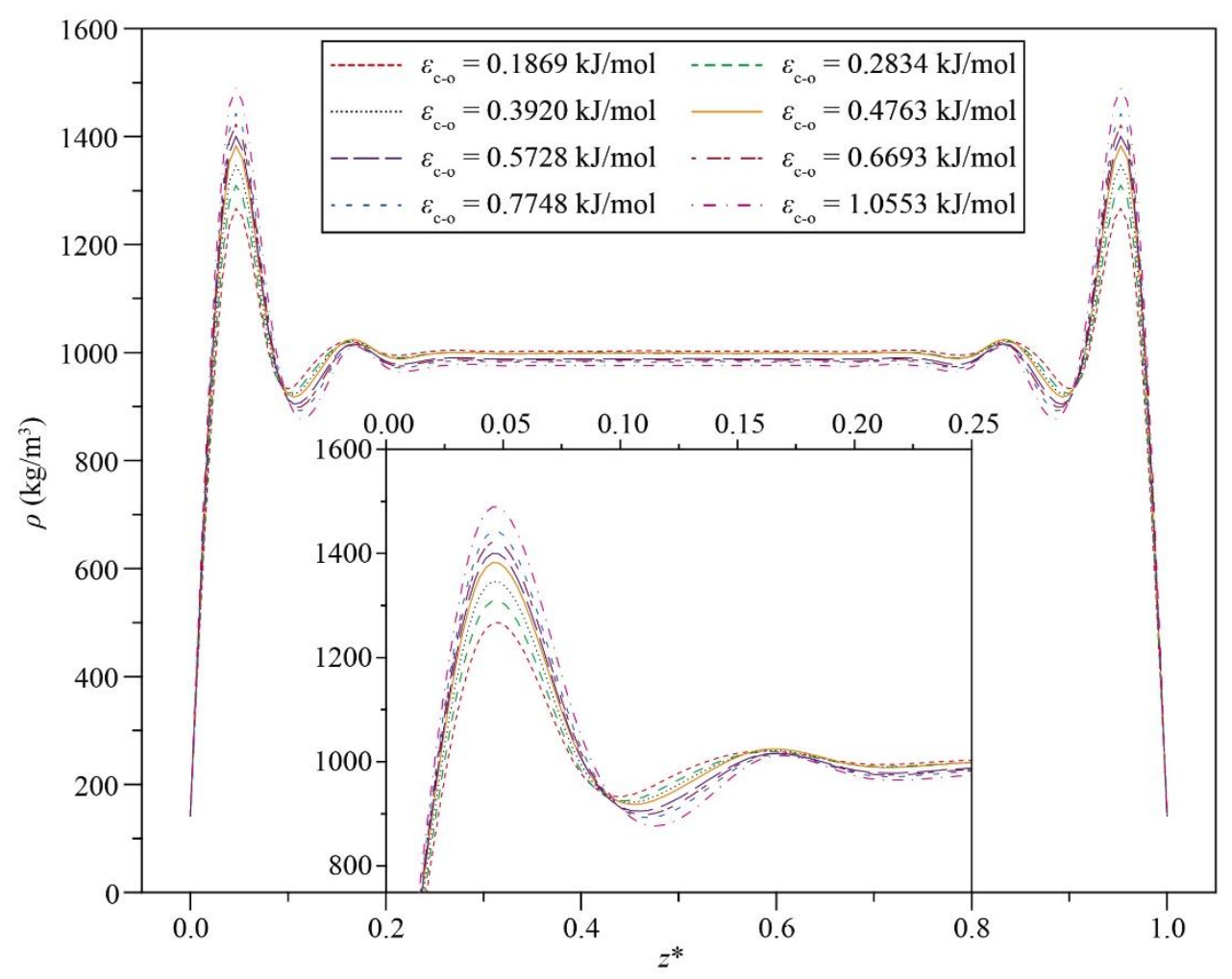

Figure 8 . Density profiles for increasing values of solid-fluid interaction strengths $\left(\varepsilon_{\mathrm{c}-\mathrm{o}}\right)$. The inset is a magnified image of density at the interfacial region.

As system size increases with channel height from $H=4.0$ to $8.0 \mathrm{~nm}$, convection performance displays enhancement with an increase towards that of macroscale behavior from $N u_{D_{f} f d}=0.97$ to 1.88 as in Fig. 7b. It is known in conventional theory that as dimensional scales increase in size within fluid flow applications, $K n$ will decrease and cease to display slip flow characteristics with trends towards that of continuum behavior. In concurrence with theory and as supported by the aforementioned $K n$ calculations, hydrodynamic slip lengths, and thus momentum at the interface, are observed to decrease as the influence of solid-fluid interfacial mechanisms weaken with increasing channel dimensions. The same is true in relation to thermal slip; at nanoscales, increasing surface-tovolume ratio permits Kapitza resistance to become a substantial factor that distorts convective performance [15]. Contrary to the previous report by Ge et al. [23] indicating nanochannel size effects produce no detectable change in heat transfer behavior, our observations indicate that as dimensions increase, interfacial mechanisms (i.e., hydrodynamic slip and thermal slip, characterizing temperature jump and Kapitza resistance) gradually become insignificant to allow for more effective heat transfer and thus a trend of convective behaviors towards that of macroscale theory. Convection heat transfer is estimated to achieve macroscale behavior for water flow in graphene nanochannels at a channel height of $32.81 \mathrm{~nm}$ and/or $605.72 \mathrm{~nm}$ using linear and logarithmic fitting, respectively.

With respect to the influence of increasing water velocity inside the nanochannel from $10 \mathrm{~m} / \mathrm{s}$ to $105 \mathrm{~m} / \mathrm{s}$, convection heat transfer behavior is observed to remain relatively constant and only deviates from $N u_{D_{f} f d}=1.23$ by $1.2 \%$ in Fig. $7 \mathrm{c}$. Conventional knowledge of nanoscale flow indicates that increasing channel velocity will yield increased slip lengths as a result of amplified momentum at the interface. Initially, it was assumed that this in turn would enable for more effective diffusion from the graphene wall to the water. However, we now suspect that insubstantial changes in momentum are not enough to produce noticeable effects on the resulting thermal diffusion within the channel. As a result, 
Kapitza resistance is left unaffected by momentum transport behaviors and produces no change in thermal performance.

As atom kinetic energy increases with thermostated wall temperature, more collisions at the wallfluid interface occur to allow for enhanced momentum and heat transfer rates; this is verified by the observance of decreases in both hydrodynamic and thermal slip lengths. Signified by the behavior of these microscopic mechanisms, this subsequent improvement in energy transfer allows for enhancement to convective behaviors inside the nanochannel with an increase from $N u_{D f f d}=1.19$ to $N u_{D, f d}=1.47$ for the thermostated wall temperatures of $T_{w}=300 \mathrm{~K}$ and $T_{w}=1000 \mathrm{~K}$, respectively (Fig. 7d). It is noted that although temperatures exceed that at which water would normally experience boiling, no phase change is observed due to the constrained simulation volume and rescaling of temperatures at the channel inlet [83].

To further validate and understand the microscopic phenomena influencing convective behaviors (Fig. 9), we investigate increasing channel heights of $H=4.0,5.0$ and $6.0 \mathrm{~nm}$ at varying carbonoxygen interaction strengths, or magnitudes of wettability, $\left(\varepsilon_{\mathrm{c}-\mathrm{o}}=0.392,0.4763\right.$ and $\left.0.5728 \mathrm{~kJ} / \mathrm{mol}\right)$ in Fig. 9a. As found above in Fig. 6, increasing interaction strength produces the most drastic changes in the resulting values of $l_{s}$ and $l_{k}$, and thus $N u_{D, f d}$. However, manipulation of $\varepsilon_{\mathrm{c}-\mathrm{o}}$ yields that at smaller channel dimensions, the effects of surface interactions are magnified and project their influence over fluid-fluid interactions within the system to induce more noticeable modifications to overall convective outcomes. Conversely, as dimensional size increases we see a lessening influence of surface mechanisms to the convective heat transfer coefficient, which is consistent with the concept that convective behaviors deviate at nanoscales due to dominating surface effects. Therefore, convection heat transfer is dependent on relative magnitudes of microscopic mechanisms (hydrodynamic and thermal slip) when compared with dimensional size.

Hydrodynamic and thermal slip $\left(l_{s}\right.$ and $\left.l_{k}\right)$ under various test conditions display good correlation (Fig. 9b) although varying $u_{f, a v g}$, which has minor influence on $l_{k}$, causes slight deviation as in the inset of Fig. 9b. This good correlation is attributed to similar control mechanisms in both interfacial momentum and thermal transport, such as strength and frequency of interactions between water molecule and surface. To evaluate the influence of size effects and these $l_{s}$ and $l_{k}$ together, $N u_{D f d}$ is plotted with respect to normalized slip lengths, $l_{s} / 2 H$ and $l_{k} / 2 H$, as respectively shown in Figs. $9 \mathrm{c}$ and $9 \mathrm{~d}$. Both collective $N u_{D, f d}$ trends are well correlated with the normalized slip lengths and indicate that larger $l_{s} / 2 H$ or $l_{k} / 2 H$ results in greater discrepancies from macroscopic convective behavior; i.e., as slip lengths increase or system size decreases, $N u_{D, f d}$ decreases. However, the correlation with $l_{s} / 2 H$ contains more deviation from the overall trend than that with $l_{k} / 2 H$; specifically, the deviation appears in the same area (dashed boxes) in Fig. $9 \mathrm{~b}$ while no such deviation and better agreement is found in the trend of $N u_{D f f d}$ with respect to $l_{k} / 2 H$. Therefore, $l_{k}$ is inferred to be the dominant factor, particularly when surface heat flux is large enough to overcome the surface advection effects imposed by slip flow as in our simulation conditions. Correlation between $N u_{D_{f} f d}$ and $l_{k} / 2 H$ is identified as the black trend line equation of $N u_{D_{f} f d}=7.544-5.595\left(1-e^{-l_{k} / 10.838 H}\right)-5.554\left(1-e^{-l_{k} / 0.2308 H}\right)$ in Fig. $9 \mathrm{~d}$; furthermore, the red trend line of $N u_{D f f d}=0.9452\left(l_{k} / 2 H\right)^{-0.7313}$ is identified as a simple fit with roughly $5 \%$ error, when $0.2<\left(l_{k} / 2 H\right)<1.2$, to estimate the macroscale value of $N u_{D, f d}$. 


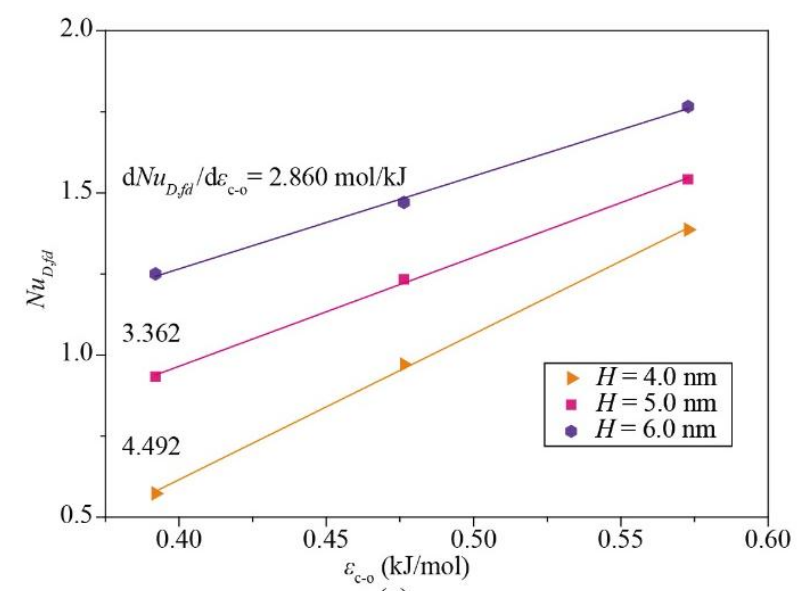

(a)

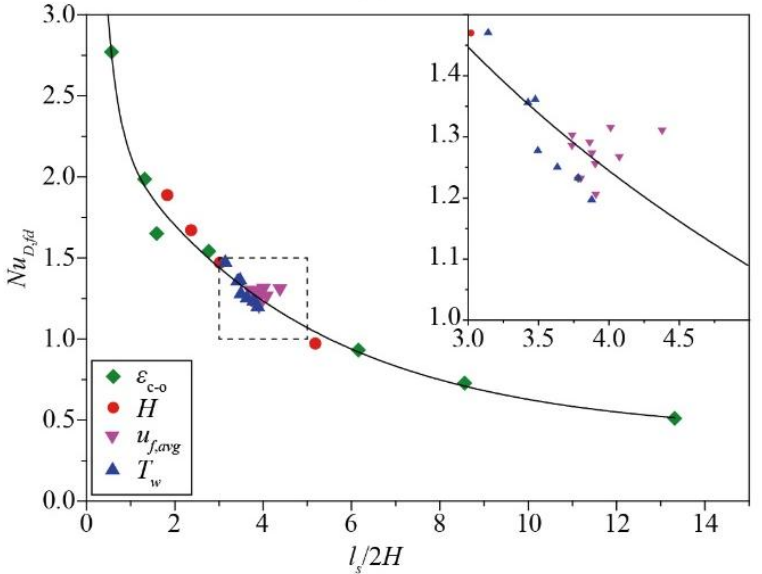

(c)



(b)



(d)

Figure 9. (a) Fully-developed $N u_{D, f d}$ trends for increasing channel heights $(H)$ at different carbon-oxygen interaction strengths $\left(\varepsilon_{\mathrm{c}-\mathrm{o}}\right)$. (b) Comparison of $l_{s}$ and $l_{k}$ for all test parameters. (c) $N u_{D_{f f d}}$ values corresponding to normalized hydrodynamic slip lengths. (d) Fully developed $N u_{D, f d}$ trend for normalized thermal slip length values.

\section{Conclusions}

Molecular dynamics simulations of water flowing in graphene nanochannels allows for investigations of the microscopic mechanisms influencing fully-developed convective heat transfer despite the presence of unrealistic axial conduction. Hydrodynamic and thermal slip are shown to be key manipulating factors in the characterization of nanoscale thermal performance. Strength and frequency of fluid-surface interactions influence both hydrodynamic and thermal slip, and thus lead to their strong correlation. However, microscopic mechanisms can be selectively controlled; for example, variation of average fluid velocity smaller than that of thermal velocity only has an effect on hydrodynamic slip (with negligible change in thermal slip). Therefore, although seemingly congruent, hydrodynamic and thermal slip are only partially related to one another as each factor possesses traits that uniquely affect convective behaviors, depending on simulation constraints. As found within our simulations, in environments where the surface heat flux is much larger than that of advection, thermal slip length is the dominating factor that governs resultant convective behavior at nanoscales. By nondimensionalizing slip lengths with system dimensions (i.e., channel height), size effects are incorporated for assessment into the relationship between $N u_{D, f d}$ and slip lengths; correlation between $N u_{D, f d}$ and $l_{k} / 2 H$ is found and suggested. 
Careful assessment of these analogous microscopic mechanisms enables for accurate description of heat transfer behaviors in system sizes descending into the nanoscale realm. Based on enhanced understandings of the mechanisms influencing and dictating nanoscale convection heat transfer within graphene-liquid water systems, this study pertains to various applications such as micro-sized heat exchangers, electric cooling systems, flow and flow boiling enhancement, primary/secondary reactor cooling loops, etc. Future work should seek to further the correlation between hydrodynamic and thermal slip, and nanoscale convection, in addition to assessment of nanochannel dimensions at which the theoretical $N u_{D f f d}$ value is obtained for optimal design and convective heat transfer performance. Additional studies should seek to evaluate the effects of graphene wall thickness on convective heat transfer similar to that of a recent study by Alexeev et al. [82]. We anticipate future assessment and validation of reported results through extrapolation of data and provided fit equations via simulation of larger dimensional scales and experiments of sub-micrometer $(<1.0 \mu \mathrm{m})$ characteristic length microchannels, similar to the methods and techniques performed by Refs. [6, 84-86].

\section{Conflict of Interest}

None declared.

\section{Acknowledgement}

This work used the Extreme Science and Engineering Discovery Environment (XSEDE), which is supported by National Science Foundation Grant number ACI-1053575. The resources of the Newton HPC Program at the University of Tennessee were also used in this work. D.M. would like to acknowledge Dr. Anton P. Markesteijn and Jiaqi Wang for their fruitful discussions and constructive insight on simulation procedures.

\section{References}

[1] A.L. Moore, L. Shi, Emerging challenges and materials for thermal management of electronics, Mater. Today 17 (4) (2014) 163-174.

[2] D.B. Tuckerman, R. Pease, High-performance heat sinking for VLSI, IEEE Electron Dev. Lett. 2

(5) (1981) 126-129.

[3] E. Pop, Energy dissipation and transport in nanoscale devices, Nano Res. 3 (3) (2010) 147-169.

[4] H. Wu, P. Cheng, Friction factors in smooth trapezoidal silicon microchannels with different aspect ratios, Int. J. Heat Mass Transf. 46 (14) (2003) 2519-2525.

[5] X. Peng, B. Wang, G. Peterson, H. Ma, Experimental investigation of heat transfer in flat plates with rectangular microchannels, Int. J. Heat Mass Transf. 38 (1) (1995) 127-137.

[6] J.M.C. Duarte, I.M.A. Contreras, C.R.C. Cely, An optimal high thermal conductive graphite microchannel for electronic devices cooling, Revista Facultad de Ingeniería (77) (2015) 143-152.

[7] S. Ghosh, I. Calizo, D. Teweldebrhan, E.P. Pokatilov, D.L. Nika, A.A. Balandin, W. Bao, F. Miao, C.N. Lau, Extremely high thermal conductivity of graphene: Prospects for thermal management applications in nanoelectronic circuits, Appl. Phys. Lett. 92 (15) (2008) 151911.

[8] A.J. Shkarah, M.Y.B. Sulaiman, M.R.B.H. Ayob, H. Togun, A 3D numerical study of heat transfer in a single-phase micro-channel heat sink using graphene, aluminum and silicon as substrates, Int. Commun. Heat Mass Transf. 48 (2013) 108-115.

[9] C.G. Stokes, On the effect of internal friction of fluids on the motion of pendulums, Trans. Camb. Philos. Soc. 9 (1851) 8-106.

[10] B. Ramos-Alvarado, S. Kumar, G.P. Peterson, Solid-Liquid Thermal Transport and Its Relationship with Wettability and the Interfacial Liquid Structure, J. Phys. Chem. Lett. 7 (17) (2016) 3497-3501.

[11] H. Brenner, Beyond the no-slip boundary condition, Phys. Rev. E 84 (4) (2011) 046309.

[12] S.-C. Yang, Effects of surface roughness and interface wettability on nanoscale flow in a nanochannel, Microfluid. Nanofluid. 2 (6) (2006) 501-511. 
[13] B.-Y. Cao, M. Chen, Z.-Y. Guo, Liquid flow in surface-nanostructured channels studied by molecular dynamics simulation, Phys. Rev. E 74 (6) (2006) 066311.

[14] L. Bocquet, J.-L. Barrat, Flow boundary conditions from nano-to micro-scales, Soft Matter. 3 (6) (2007) 685-693.

[15] B.-Y. Cao, J. Sun, M. Chen, Z.-Y. Guo, Molecular momentum transport at fluid-solid interfaces in MEMS/NEMS: a review, Int. J. Mol. Sci. 10 (11) (2009) 4638-4706.

[16] T.Q. Vo, B. Kim, Transport Phenomena of Water in Molecular Fluidic Channels, Sci. Rep. 6 (2016) 33881.

[17] T.A. Ho, D.V. Papavassiliou, L.L. Lee, A. Striolo, Liquid water can slip on a hydrophilic surface, Proc. Nat. Acad. Sci. U. S. A. 108 (39) (2011) 16170-16175.

[18] J.A. Thomas, A.J. McGaughey, Reassessing fast water transport through carbon nanotubes, Nano Lett. 8 (9) (2008) 2788-2793.

[19] J.A. Thomas, A.J. McGaughey, O. Kuter-Arnebeck, Pressure-driven water flow through carbon nanotubes: insights from molecular dynamics simulation, Int. J. Therm. Sci. 49 (2) (2010) 281-289.

[20] C. Soong, T. Yen, P. Tzeng, Molecular dynamics simulation of nanochannel flows with effects of wall lattice-fluid interactions, Phys. Rev. E 76 (3) (2007) 036303.

[21] Y.A. Gandomi, M. Edmundson, F. Busby, M.M. Mench, Water Management in Polymer Electrolyte Fuel Cells through Asymmetric Thermal and Mass Transport Engineering of the MicroPorous Layers, J. Electrochem. Soc. 163 (8) (2016) F933-F944.

[22] W. Qu, G.M. Mala, D. Li, Heat transfer for water flow in trapezoidal silicon microchannels, Int. J. Heat Mass Transf. 43 (21) (2000) 3925-3936.

[23] S. Ge, Y. Gu, M. Chen, A molecular dynamics simulation on the convective heat transfer in nanochannels, Mol. Phys. 113 (7) (2015) 703-710.

[24] J.F. Thekkethala, S.P. Sathian, The effect of graphene layers on interfacial thermal resistance in composite nanochannels with flow, Microfluid. Nanofluid. 18 (4) (2015) 637-648.

[25] J.-L. Barrat, F. Chiaruttini, Kapitza resistance at the liquid — solid interface, Mol. Phys. 101 (11) (2003) 1605-1610.

[26] H. Ghasemi, C. Ward, Mechanism of sessile water droplet evaporation: kapitza resistance at the solid-liquid interface, J. Phys. Chem. C 115 (43) (2011) 21311-21319.

[27] C. Bakli, S. Chakraborty, Slippery to sticky transition of hydrophobic nanochannels, Nano Lett. 15 (11) (2015) 7497-7502.

[28] B.H. Kim, A. Beskok, T. Cagin, Viscous heating in nanoscale shear driven liquid flows, Microfluid. Nanofluid. 9 (1) (2010) 31-40.

[29] H. Ghasemi, C. Ward, Mechanism of sessile water droplet evaporation: kapitza resistance at the solid-liquid interface, J. Phys. Chem. C 115 (43) (2011) 21311-21319.

[30] J. Towns, T. Cockerill, M. Dahan, I. Foster, K. Gaither, A. Grimshaw, V. Hazlewood, S.

Lathrop, D. Lifka, G.D. Peterson, XSEDE: accelerating scientific discovery, Comput. Sci. Eng. 16 (5) (2014) 62-74.

[31] J.L. Abascal, C. Vega, A general purpose model for the condensed phases of water: TIP4P/2005, J. Chem. Phys. 123 (23) (2005) 234505.

[32] C. Vega, J.L. Abascal, Simulating water with rigid non-polarizable models: a general perspective, Phys. Chem. Chem. Phys. 13 (44) (2011) 19663-19688.

[33] J.-P. Ryckaert, G. Ciccotti, H.J. Berendsen, Numerical integration of the cartesian equations of motion of a system with constraints: molecular dynamics of n-alkanes, J. Comput. Phys. 23 (3) (1977) 327-341.

[34] M. Moulod, G. Hwang, Water self-diffusivity confined in graphene nanogap using molecular dynamics simulations, J. Appl. Phys. 120 (19) (2016) 194302.

[35] G. Cicero, J.C. Grossman, E. Schwegler, F. Gygi, G. Galli, Water confined in nanotubes and between graphene sheets: A first principle study, J. Am. Chem. Soc. 130 (6) (2008) 1871-1878. [36] J. Marti, J. Sala, E. Guardia, Molecular dynamics simulations of water confined in graphene nanochannels: From ambient to supercritical environments, J. Mol. Liq. 153 (1) (2010) 72-78. [37] D.W. Brenner, O.A. Shenderova, J.A. Harrison, S.J. Stuart, B. Ni, S.B. Sinnott, A secondgeneration reactive empirical bond order (REBO) potential energy expression for hydrocarbons, J. Phys.: Condens. Matter. 14 (4) (2002) 783. 
[38] L. Girifalco, M. Hodak, R.S. Lee, Carbon nanotubes, buckyballs, ropes, and a universal graphitic potential, Phys. Rev. B 62 (19) (2000) 13104.

[39] M. Gordillo, J. Mart1, Hydrogen bond structure of liquid water confined in nanotubes, Chem. Phys. Lett. 329 (5) (2000) 341-345.

[40] J. Rafiee, X. Mi, H. Gullapalli, A.V. Thomas, F. Yavari, Y. Shi, P.M. Ajayan, N.A. Koratkar, Wetting transparency of graphene, Nature Mater. 11 (3) (2012) 217-222.

[41] R. Raj, S.C. Maroo, E.N. Wang, Wettability of graphene, Nano Lett. 13 (4) (2013) 1509-1515. [42] Z. Li, Y. Wang, A. Kozbial, G. Shenoy, F. Zhou, R. McGinley, P. Ireland, B. Morganstein, A. Kunkel, S.P. Surwade, Effect of airborne contaminants on the wettability of supported graphene and graphite, Nature Mater. 12 (10) (2013) 925-931.

[43] B. Ramos-Alvarado, S. Kumar, G. Peterson, Wettability of graphitic-carbon and silicon surfaces: MD modeling and theoretical analysis, J. Chem. Phys. 143 (4) (2015) 044703.

[44] B. Ramos-Alvarado, S. Kumar, G. Peterson, Hydrodynamic slip length as a surface property, Phys. Rev. E 93 (2) (2016) 023101.

[45] L. Verlet, Computer" experiments" on classical fluids. I. Thermodynamical properties of Lennard-Jones molecules, Phys. Rev. 159 (1) (1967) 98.

[46] R.W. Hockney, J.W. Eastwood, Computer simulation using particles, CRC Press, 1988.

[47] E. Spohr, Effect of electrostatic boundary conditions and system size on the interfacial properties of water and aqueous solutions, J. Chem. Phys. 107 (16) (1997) 6342-6348.

[48] Y. Mao, Y. Zhang, Thermal conductivity, shear viscosity and specific heat of rigid water models, Chem. Phys. Lett. 542 (2012) 37-41.

[49] National Institute of Standards and Technology, NIST Chemistry Webbook, http://webbook.nist.gov/chemistry/.

[50] R.F. Probstein, Physicochemical Hydrodynamics: An Introduction, John Wiley \& Sons, New York, 2005.

[51] K.V. Sharp, R.J. Adrian, J.G. Santiago, J.I. Molho, The MEMS Handbook, CRC, New York, (2002) 1-33.

[52] S. Kandlikar, S. Garimella, D. Li, S. Colin, M.R. King, Heat Transfer and Fluid Flow in Minichannels and Microchannels, Elsevier, Amsterdam, 2006.

[53] M. Gad-el-Hak, Differences between liquid and gas transport at the microscale, Bull. Pol. Acad. Sci. 53 (4) (2005) 301-316.

[54] K.P. Travis, B. Todd, D.J. Evans, Departure from Navier-Stokes hydrodynamics in confined liquids, Phys. Rev. E 55 (4) (1997) 4288.

[55] K.P. Travis, K.E. Gubbins, Poiseuille flow of Lennard-Jones fluids in narrow slit pores, J. Chem. Phys. 112 (4) (2000) 1984-1994.

[56] X.-B. Mi, A.T. Chwang, Molecular dynamics simulations of nanochannel flows at low Reynolds numbers, Molecules 8 (1) (2003) 193-206.

[57] J. Lahjomri, A. Oubarra, Analytical solution of the Graetz problem with axial conduction, J. Heat Transf. 121 (4) (1999) 1078-1083.

[58] Y. Haddout, J. Lahjomri, The extended Graetz problem for a gaseous slip flow in micropipe and parallel-plate microchannel with heating section of finite length: Effects of axial conduction, viscous dissipation and pressure work, Int. J. Heat Mass Transf. 80 (2015) 673-687.

[59] A. Markvoort, P. Hilbers, S. Nedea, Molecular dynamics study of the influence of wall-gas interactions on heat flow in nanochannels, Phys. Rev. E 71 (6) (2005) 066702.

[60] S. Nosé, A molecular dynamics method for simulations in the canonical ensemble, Mol. Phys. 52

(2) (1984) 255-268.

[61] W.G. Hoover, Canonical dynamics: equilibrium phase-space distributions, Phys. Rev. A 31 (3) (1985) 1695.

[62] T. Schneider, E. Stoll, Dynamics of the Sine-Gordon Chain, Phys. Rev. Lett. 41 (21) (1978) 1429.

[63] D.C. Rapaport, The art of molecular dynamics simulation, Cambridge University Press, 2004. [64] R. Khare, P. Keblinski, A. Yethiraj, Molecular dynamics simulations of heat and momentum transfer at a solid-fluid interface: relationship between thermal and velocity slip, Int. J. Heat Mass Transf. 49 (19) (2006) 3401-3407. 
[65] R.K. Shah, A.L. London, Laminar Flow Forced Convection in Ducts, Academic Press, New York, 1978.

[66] F.P. Incropera, D.P. Dewitt, T.L. Bergman, A.S. Lavine, Fundamentals of Heat and Mass Transfer, sixth ed., John Wiley \& Sons, Inc., Hoboken, New Jersey, 2007.

[67] T. Werder, J. Walther, R. Jaffe, T. Halicioglu, P. Koumoutsakos, On the water-carbon interaction for use in molecular dynamics simulations of graphite and carbon nanotubes, J. Phys. Chem. B 107

(6) (2003) 1345-1352.

[68] Y.-W. Gu, S. Ge, M. Chen, A molecular dynamics simulation of nanoscale convective heat transfer with the effect of axial heat conduction, Mol. Phys. 114 (12) (2016) 1922-1930.

[69] A. Markesteijn, R. Hartkamp, S. Luding, J. Westerweel, A comparison of the value of viscosity for several water models using Poiseuille flow in a nano-channel, J. Chem. Phys. 136 (13) (2012) 134104.

[70] S. Shin, M. Kaviany, T. Desai, R. Bonner, Roles of atomic restructuring in interfacial phonon transport, Phys. Rev. B 82 (8) (2010) 081302.

[71] S.K. Kannam, B. Todd, J.S. Hansen, P.J. Daivis, Slip length of water on graphene: Limitations of non-equilibrium molecular dynamics simulations, J. Chem. Phys. 136 (2) (2012) 024705.

[72] S.K. Kannam, B. Todd, J.S. Hansen, P.J. Daivis, Slip flow in graphene nanochannels, J. Chem. Phys. 135 (14) (2011) 144701.

[73] P.A. Thompson, S.M. Troian, A general boundary condition for liquid flow at solid surfaces, Nature 389 (6649) (1997) 360-362.

[74] C. Sendner, D. Horinek, L. Bocquet, R.R. Netz, Interfacial water at hydrophobic and hydrophilic surfaces: Slip, viscosity, and diffusion, Langmuir 25 (18) (2009) 10768-10781.

[75] T.Q. Vo, B. Kim, Interface thermal resistance between liquid water and various metallic surfaces, Int. J. Precis. Eng. Manuf. 16 (7) (2015) 1341-1346.

[76] L. Xue, P. Keblinski, S. Phillpot, S.-S. Choi, J. Eastman, Two regimes of thermal resistance at a liquid-solid interface, J. Chem. Phys. 118 (1) (2003) 337-339.

[77] X. Yang, Z.C. Zheng, Effects of channel scale on slip length of flow in micro/nano channels, ASME J. Fluids Eng. 132 (2010) 061201.

[78] R.S. Voronov, D.V. Papavassiliou, L.L. Lee, Boundary slip and wetting properties of interfaces: correlation of the contact angle with the slip length, J. Chem. Phys. 124 (20) (2006) 204701.

[79] A. Martini, H.-Y. Hsu, N.A. Patankar, S. Lichter, Slip at high shear rates, Phys. Rev. Lett. 100

(20) (2008) 206001.

[80] A.A. Pahlavan, J.B. Freund, Effect of solid properties on slip at a fluid-solid interface, Phys. Rev. E 83 (2) (2011) 021602.

[81] M. Barisik, A. Beskok, Temperature dependence of thermal resistance at the water/silicon interface, Int. J. Therm. Sci. 77 (2014) 47-54.

[82] D. Alexeev, J. Chen, J.H. Walther, K.P. Giapis, P. Angelikopoulos, P. Koumoutsakos, Kapitza resistance between few-layer graphene and water: Liquid layering effects, Nano Lett. 15 (9) (2015) 5744-5749.

[83] V.V. Chaban, O.V. Prezhdo, Water boiling inside carbon nanotubes: toward efficient drug release, ACS Nano 5 (7) (2011) 5647-5655.

[84] S.-S. Hsieh, C.-Y. Lin, Convective heat transfer in liquid microchannels with hydrophobic and hydrophilic surfaces, Int. J. Heat Mass Transf. 52 (1) (2009) 260-270.

[85] B. Kim, An experimental study on fully developed laminar flow and heat transfer in rectangular microchannels, Int. J. Heat Fluid Flow 62 (2016) 224-232.

[86] I. Silvestre, A.W. Barnard, S.P. Roberts, P.L. McEuen, R.G. Lacerda, Folded graphene nanochannels via pulsed patterning of graphene, Appl. Phys. Lett. 106 (15) (2015) 153105. 